\title{
A Pilot Study: Is Snoring During Pediatric Dental Procedures Indicative of Obstructive Sleep Apnea?
}

\author{
Harmeet K. Chiang, DDS, MS¹; Riley J. Robinson, BS¹; Al M. Best, PhD¹; Tegwyn H. Brickhouse, DDS, PhD²; David J. \\ Leszczyszyn, MD, $\mathrm{PhD}^{3}$
}

\begin{abstract}
${ }^{1}$ School of Dentistry, Virginia Commonwealth University, Richmond, VA; ${ }^{2}$ Department of Oral Health Promotion and Community Outreach, Virginia Commonwealth University, Richmond, VA; ${ }^{3}$ Center for Sleep Medicine, Virginia Commonwealth University, Richmond, VA;
\end{abstract}

\begin{abstract}
Study Objectives: To determine whether pediatric snoring during sedation could be used to screen for obstructive sleep apnea (OSA) by pediatric dentists.

Methods: Pediatric dental patients requiring moderate sedation with American Society of Anesthesiologists (I or II) or Brodsky classification (grade 1 and 2) were identified, recruited, separated in groups of snorers and nonsnorers, and then matched based on body mass index to within $\pm 5 \%$. Parents completed a Pediatric Symptom Checklist (PSC), and Pediatric Sleep Questionnaire (PSQ). OSA was diagnosed using a home sleep monitoring device. Groups were compared using a t-test.

Results: Overall, 19 children were screened for eligibility and 17 enrolled: 10 who snored (59\%) and 7 control participants. Although children who snored during sedation had a nominally higher respiratory disturbance index $(\mathrm{RDI})(\mathrm{mean}=5.3 \mathrm{vs}$. 4.3$)$, this difference was not statistically significant ( $p>0.7$ ). Using a cutoff RDI $>5$ to indicate significant OSA, 5 of 8 in the snorer group and 2 of 4 in the nonsnorer group met this criterion. Conversely, one nonsnoring participant had a maximum relative loudness of $27 \mathrm{~dB}$, which resulted in a nominally higher group mean than the snoring group. The questionnaire results of both the PSC and PSQ average was 5 points higher in the snoring group but the difference was not statistically significant (PSQ $p=0.056$, PSC $p>0.4$ ).

Conclusions: Snoring alone during pediatric dental procedures does not appear to be indicative of obstructive sleep apnea. Recognition of pediatric sleep apnea may be enhanced through questionnaires and adding snore analysis to home-based sleep apnea screening.

Keywords: moderate sedation, obstructive sleep apnea, OSA, pediatric dentist, screening

Citation: Chiang HK, Robinson RJ, Best AM, Brickhouse TH, Leszczyszyn, DJ. A Pilot Study: Is Snoring During Pediatric Dental Procedures Indicative of Obstructive Sleep Apnea?. J Dent Sleep Med. 2019;6(2)
\end{abstract}

\section{INTRODUCTION}

Obstructive sleep apnea (OSA), also referred to as obstructive sleep apnea/hypopnea syndrome, is a sleep disorder characterized by obstructive apneas and hypopneas that occur due to hindrance in the upper airway resulting in periodic obstruction in the respiratory gas exchange, eventually leading to sleep interruption. ${ }^{1,2}$ Additionally, OSA associated with excessive daytime sleepiness is commonly referred to as obstructive sleep apnea syndrome (OSAS). For the purposes of continuity in this study, both OSA and OSAS will be referred to as OSA.

Individuals of all ages are affected by OSA, with increased prevalence among pediatric and adolescent populations. ${ }^{3}$ Childhood OSA is one of the most common, unrecognized, under-reported, underdiagnosed chronic diseases. ${ }^{3}$ OSA in adults is defined as apnea that lasts more than 10 seconds, and this is often associated with snoring.1 In children, apneas may last no more than 3 to 4 seconds ( 2 missed breaths), and these have clearly been associated with oxygen desaturation. Such witnessed apneas have been reported in $5 \%$ of children (range $0.5 \%$ to $9 \%$ ). OSA affects $1 \%$ to $10 \%$ of children, whereas $3 \%$ to $12 \%$ of children snore. ${ }^{4,5}$ Studies show that older children are less likely to snore than younger children. OSA has been estimated to affect $2.4 \%$ of all children age 2 to 8 years and approximately 500,000 children in the United States overall. ${ }^{6,7}$

The causes of OSA are categorized into (1) anatomical anomalies: hypertrophic tonsils or adenoids (most common in children), (2) neuromuscular diseases, and (3) craniofacial anomalies or syndromes. ${ }^{8}$ Other than actually observing apnea or cyanosis, children snoring at night is the most important indicator of predicting OSA. ${ }^{2,9}$ Other symptoms of pediatric OSA, which could differ from those in adults, include longer total sleep time than normal, more effort expended during breathing (flaring nostrils, heaving chests, sweating), and behavioral difficulties without any obvious cause, such as hyperactivity and inattention, irritability, bedwetting, morning headaches, failure to grow, and weight gain. ${ }^{10}$ If OSA is undiagnosed, it may lead to severe developmental problems, affect neural behavioral issues or cognitive function, and can be associated with failure to thrive, enuresis, attention deficit hyperactivity disorder, poor academic performance, and cardiopulmonary diseases. ${ }^{4,8}$ Children with OSA are $226 \%$ more likely than children without OSA to require health care. ${ }^{7}$ Cases of respiratory failure or coma (with few cases of death) in children have been reported. ${ }^{11}$

The American Academy of Pediatrics Clinical 
Practice Guideline recommends early screening, diagnosis, and treatment as the key to mitigating the long-term effects of OSA. Identification and treatment can result in alleviation of current symptoms, improved quality of life, prevention of sequelae, education of parents, and decreased health care utilization. ${ }^{11}$

Polysomnography (PSG) is considered the gold standard to diagnose OSA. PSG records apnea and hypopnea events during sleep. OSA is ultimately diagnosed by measuring patients' apnea-hypopnea index (AHI), which measures the severity of OSA. AHI is measured as the number of apneas and hypopneas per hour of sleep. The clinical importance of a child's OSA severity is typically determined by compiling their presenting clinical symptoms and weighing the patients' AHI value from a laboratory-based polysomnogram, or the related respiratory disturbance index (RDI) from a home-based polysomnogram. RDI is measured as the number of apneas, hypopneas, and respiratory effort-related arousals (RERAs) per hour of sleep. RERAs are considered sleep disruptions not classified as an apnea or hypopnea. The AHI and RDI are considered the gold standard in the diagnosis of OSA. Mild pediatric OSA is defined as an AHI or RDI from 1 to 4.9 events/h, whereas severe OSA is defined as an AHI or RDI that exceeds 10 events/h. ${ }^{12}$

Moderate OSA criteria in children is defined by an RDI or AHI from 5 to 9.9 events/h, a level at which most clinicians would recommend active intervention even in the absence of comorbid medical disease or daytime neurobehavioral symptoms. This study defined clinically significant OSA at this same level--meeting the RDI definition of moderate or greater severity - because the recruited children had either no snoring symptoms or snoring was present only as an isolated symptom.

Because laboratory-based PSG is only performed in an overnight clinical setting, this diagnostic method may not be practical for many patients because of the limited number of sleep laboratories. Home-based options are simplified, performed in the person's natural sleep environment, and eliminate an access-to-treatment barrier as no wait time is notable and equipment is more readily available. Reported sensitivities for the home-based studies range from $86 \%$ to $100 \%$, whereas specificities range from $64 \%$ to $100 \%$. The reported coefficient of correlation for AHI between two tests ranged from 0.74-0.98. ${ }^{13}$ Additionally, the use of laboratory-based PSG as a diagnostic tool to discriminate primary snorers from children suffering from OSA is not cost effective. ${ }^{5,7}$ For these reasons, an in-home sleep test (SNAP, Snap Diagnostics, LLC, Wheeling, IL, USA) was utilized. Therefore, measurements will be reported in RDI.

An important limitation in clinical screening tests such as logarithmic equations, cephalometry, morphometry studies, and other clinical tests is that they may produce mixed results. ${ }^{14} \mathrm{~A}$ recent meta-analysis of clinical tools for screening of OSA in adults concluded that current questionnaires and clinical models may miss a significant proportion of patients with OSA.${ }^{14}$ However, a recent systematic review of diagnostic tools in children indicated that three different snoring indicators were important components for identifying sleep disorders: snoring that disturbs others, snoring every night, and snoring extremely loudly. ${ }^{9}$ Therefore, general medical history interviews should include questionnaires that ask simple questions about snoring frequency and loudness, witnessed nocturnal apneas, and daytime sleepiness. Other than snoring, oral and craniofacial consequences ${ }^{9}$ as well as accurate clinical symptoms such as observed apnea, mouth breathing, excessive daytime somnolence, and large tonsil size are most commonly used to diagnose OSA. ${ }^{8}$ Recognition of these factors in patients that report frequent symptoms of OSA should lead to a referral for further OSA evaluation. ${ }^{5}$

Prior to a referral for a PSG diagnosis, several screening tools for OSA have been proposed and most include an assessment of snoring. Almost all children with OSA snore, and almost no episodes of obstructive apneas and hypopneas are observed in nonsnoring healthy children. Thus, snoring may be used as a screening symptom for recognizing OSA. ${ }^{11,16}$

Although most patients with OSA snore, not all snorers have OSA. Therefore, it is important to incorporate simple clinical tests with a low false-positive rate. A recent prospective study found that the indices most predictive of OSA in adults are snoring severity combined with body mass index (BMI) and that increased snoring duration and loudness are associated with increased severity of OSA. ${ }^{17}$ In the absence of OSA, increased snoring is associated with oxygen desaturations. Similarly, pediatric snoring is objectively related to OSA severity. ${ }^{18}$ These clinical assessments may be useful in risk stratifying of patients for PSG and therapy, facilitating deferred workup in low-risk patients, and expedited therapy in high-risk patients. ${ }^{17}$

Because the American Academy of Pediatrics strongly recommends incorporating snoring as a screening tool during a child's routine maintenance visits, pediatric dentists could use snoring as a sensitive predictor in early identification of OSA, which would provide additional clinical indicators that can complement the current diagnostic and screening tools to recognize OSA symptoms. ${ }^{9,19}$ Authors have indicated that an average delay of 23 months occurred between identification of pediatric patients with large tonsils and their referral to a sleep clinic. ${ }^{8}$ Early identification of OSA is desirable, because it is a high prevalence condition, and identification and treatment can result in alleviation of current symptoms, improved quality of life, prevention of sequelae, education of parents, and decreased health care utilization. ${ }^{11}$ If a child can be identified as a snorer or displays symptoms of OSA, clinicians will have an earlier occasion to conduct a more focused evaluation for treatment wherein an AHI or RDI and a snoring index (SI) (snores per hour) can be 
recorded..$^{11} \mathrm{SI}$ is recorded as the number of snoring events per hours of sleep.

Pediatric dentists routinely use moderate sedation to manage pediatric dental patients and see the children frequently for dental care. Medications used during sedation aggravate OSA by altering the normal mechanism of respiration through decreasing pharyngeal muscle tone, depressing ventilatory responses to hypoxia and hypercapnia; and in turn depressing arousal responses. ${ }^{19}$ Therefore, pediatric dentists have a unique opportunity to identify such snoring children and aid in the early detection of OSA. ${ }^{8,9}$ A national survey of American Association of Pediatric Dentists members estimated that in a typical year more than 308,448 pediatric patients receive treatment under sedation and could be screened for OSA. ${ }^{20}$ Sharma et al. reported that $74 \%$ of patients (17 of 23) with previously unrecognized OSA undergoing various diagnostic procedures under moderate sedation (MS) received a diagnosis of OSA. ${ }^{21}$ Additionally, Sharara et al. reported that in a single-center study of 131 adult patients, 24 snored while under MS during a colonoscopy and were referred for PSG. Of these 24 patients, 20 agreed to proceed with PSG and all had evidence of OSA, which was found to be moderate to severe in 14 of 20 patients. ${ }^{19}$ Because monitoring for snoring while under MS is suggested to be an important screening tool for adults during a colonoscopy, this study aims to investigate the role that monitoring while under MS during dental procedures could play in the pediatric population.

Pediatric dental offices could serve as a site for detection and identification of OSA signs during moderate sedation procedures. The purpose of this pilot study is to examine a simple objective measure of pediatric snoring as a screening tool for risk stratification of OSA in pediatric patients undergoing moderate sedation for dental procedures.

To the best of our knowledge, this is the first study to examine the prevalence of previously undiagnosed OSA associated with snoring in pediatric patients under moderate sedation (MS). This is the first study to offer an objective definition for "significant (audible) snoring." A challenge in OSA screening is that reported snoring and apnea frequency is subject to large variations due to various cultural factors and perceptions that gauge the relative loudness or noisy breathing as "snoring."

\section{METHODS}

This pilot case-control study was approved by the Virginia Commonwealth University (VCU) Institutional Review Board (IRB: \#HM15315). Pediatric patients in need of dental procedures requiring moderate sedation were screened to be eligible for inclusion in this study. Pediatric patients were not specially sedated for the purpose of this study and normal sedation protocol was followed. Patients were required to have an American
Society of Anesthesiologists (ASA) classification of I or II and a Brodsky grade of 1 or 2 (tonsil size less than 50\%).

Parents/guardians of eligible patients meeting these requirements were approached to consent to the study to be monitored for snoring during moderate sedation and the associated recovery time, and complete a Pediatric Symptom Checklist (PSC) ${ }^{15}$ and a one-page sleep-related breathing disorder (SRBD) scale consisting of 22 closed response question-items extracted from the Pediatric Sleep Questionnaire (PSQ). ${ }^{22}$ The PSC requires a minimum of 31 responses from 35 items. If more than four answers are left blank, the questionnaire is considered invalid. The items are rated as "Never," "Sometimes," or "Often" present. These responses are scored 0,1 , and 2 , respectively. The total score is calculated by adding the scores from each of the 35 items. ${ }^{23}$ Possible responses to the 22 questions in the PSQ (SRBD) are "yes"= 1, "no"=0, and "don't know"= missing. The mean score is calculated from responses of "yes" and "no." The cutoff value of 0.33 is most effective in identifying pediatric OSA.24 The SRBD scale is designed to screen SRBD and associated symptom when PSG is not feasible. The SRBD includes a snoring subscale. ${ }^{19}$ Both the PSQ (SRBD scale) and PSC are validated screening tools to assess pediatric sleep disorder symptoms and behavioral symptoms of OSA. ${ }^{15,22,25}$ The PSC behavioral symptom survey was used to pick up any mild forms of OSA that may only present with behavioral symptoms. ${ }^{15}$

During the moderate sedation procedure and the associated recovery time, snoring was noted and recorded by the pediatric dentist to classify patients as snorers and nonsnorers for grouping. A somewhat objective measure of "significant (audible) snoring" while under sedation for the dental procedure was used. Specifically, "significant (audible) snoring" was defined as turbulent sounds lasting as long as or longer than two breaths. Fleming et al. ${ }^{26}$ described the respiratory rate (breaths per minute) of children for specific ages. For the calibration purposes of this study, the respiratory rates were converted to seconds per breath and it was noticed that 10 seconds in the oldest children corresponded to the 10th percentile of two breaths (Table 1). ${ }^{26}$ As two breaths is the same length of time used to define an apnea or hypopnea, the 10th percentile of the respiratory rate for age was used as a cutoff for "significant audible snoring."

The snorers and nonsnorers were identified, matched by their BMI percentile to within $\pm 5 \%$, and asked to participate in an additional study which required the pediatric patient to wear the SNAP sleep device at home for 1 night to definitively diagnose OSA by recording apneas, hypopneas, and RERAs. No additional risks to pediatric patients were involved in the sleep study. Additionally, a referral was provided to patients who tested positive for OSA. Participants were permitted to withdraw from the study at any time, including withdrawal if the child could not tolerate the SNAP device. 
Table 1. Number of Seconds for two missed breaths by Percentiles of respiratory rate for normal children from birth to 18 years of age

\begin{tabular}{llllllll} 
& \multicolumn{7}{c}{ Two missed breaths longer than (percentiles) } \\
\cline { 2 - 8 } Age range & $1 \mathrm{st}$ & 10 th & 25 th & Median & 75th & 90th & 99th \\
\hline $0-3 \mathrm{~m}$ & 4.8 & 3.5 & 3.0 & 2.8 & 2.3 & 2.1 & 1.8 \\
$3-6 \mathrm{~m}$ & 5.0 & 3.6 & 3.2 & 2.9 & 2.4 & 2.2 & 1.9 \\
$6-9 \mathrm{~m}$ & 5.2 & 3.9 & 3.3 & 3.1 & 2.6 & 2.3 & 2.0 \\
$9-12 \mathrm{~m}$ & 5.5 & 4.0 & 3.4 & 3.2 & 2.7 & 2.4 & 2.1 \\
$12-18 \mathrm{~m}$ & 5.7 & 4.3 & 3.8 & 3.4 & 2.9 & 2.6 & 2.3 \\
$18-24 \mathrm{~m}$ & 6.3 & 4.8 & 4.1 & 3.9 & 3.3 & 3.0 & 2.6 \\
$2-3 \mathrm{y}$ & 6.7 & 5.5 & 4.8 & 4.3 & 3.9 & 3.5 & 3.2 \\
$3-4 \mathrm{y}$ & 7.1 & 5.7 & 5.2 & 4.8 & 4.4 & 4.1 & 3.6 \\
$4-6 \mathrm{y}$ & 7.1 & 6.0 & 5.7 & 5.2 & 4.8 & 4.4 & 4.1 \\
$6-8 \mathrm{y}$ & 7.5 & 6.7 & 6.0 & 5.7 & 5.2 & 5.0 & 4.4 \\
$8-12 \mathrm{y}$ & 8.6 & 7.5 & 6.7 & 6.3 & 5.7 & 5.5 & 4.8 \\
$12-15 \mathrm{y}$ & 10.0 & 8.0 & 7.5 & 6.7 & 6.3 & 5.7 & 5.2 \\
$15-18 \mathrm{y}$ & 10.9 & 9.2 & 8.0 & 7.5 & 6.7 & 6.3 & 5.5 \\
\hline
\end{tabular}

This study utilized a portable SNAP device to diagnose OSA instead of performing a laboratory-based PSG. The SNAP diagnostic device received FDA approval for off-site sleep testing service in 1996 and is accepted for use on patients as young as 2 years. Over the past 20 years, the SNAP monitor has been used in 25,000 pediatric studies. There is a statistically significant Pearson correlation coefficient $(r=0.916)$ between the AHIs determined from PSG and RDIs determined from SNAP. ${ }^{27}$ SNAP is an acceptable alternative OSA testing modality to PSG and is associated with less expense, decreased waiting time, and increased convenience. ${ }^{27,28}$

Following eligibility screening, recruitment, and grouping identification, information was acquired and analyzed from three sources: (1) parental questionnaires, (2) pediatric dentistry sedation records, and (3) the SNAP pediatric portable sleep monitoring device. The two PSQ (SRBD scale) and PSC questionnaires were used. From the Sedation Record, the following information was extracted: sex, age, weight, snoring/OSA, ASA classification, and airway assessment (obesity, limited neck mobility, micrognathia/retrognathia, tonsillar obstruction $\%$, upper airway clear, lungs clear). As an addendum to the sedation record, an additional sheet recorded: race, BMI, types of airway interventions (nasal pharyngeal airway, suctioning of airway, oral pharyngeal airway, shoulder roll, chin lift), snoring criteria, snoring events, and included a table to track the snoring events throughout the procedure. The following pediatric portable sleep monitoring device (SNAP) parameters were recorded: oral nasal cannula connected to a microphone recorded oronasal airflow and oronasal sound, pulse oximetry, and heart rate and respiratory effort recorded with a chest belt and a recording unit. Not all the sleep-related indices were obtainable from all the participants but all that were obtained were used in the analyses. These measures were used for diagnosis. Sleep study data were reviewed by a sleep specialist, blinded to subject information, for quality and scoring. The outcome was recorded as the PSQ and PSC calculated from the questionnaires, participant characteristic information from the pediatric sedation record, RDI classified as normal/mild/moderate or severe, ${ }^{9} \mathrm{SI},(\%)$ Type $1 \& 2$, and Oro Sound Max Rel. dB from SNAP diagnostics. SNAP diagnostics identified and profiled all snoring events into five categories. Type 1 and type 2 snoring represent velumlike snoring (palatal snoring), which was recorded and analyzed as this type is associated with increased severity of OSA. ${ }^{17}$ Maximum relative loudness was recorded as the average loudness of the loudest $10 \%$ of all snoring events. The average relative loudness is the average of all snoring events with snoring ranges classified as mild $(0-10 \mathrm{~dB})$, moderate $(10-20 \mathrm{~dB})$, and severe $(>20 \mathrm{~dB})$. SNAP snoring loudness is measured relative to the sound of normal breathing at the opening of the mouth/nose and where $0 \mathrm{~dB}$ in the unit is equivalent to approximately $65 \mathrm{~dB}$ Sound Pressure Level (SPL).

All statistical analyses were performed using SAS software (JMP pro version 11, SAS version 9.3, SAS Institute Inc., Cary, NC, USA). Sample size calculations were done using nQuery Advisor software (Statistical Solutions Ltd, Cork, Ireland). Specifically, the estimated prevalence including 95\% confidence interval was assessed regarding percentage of children who snored under moderate sedation, the percentage of those who snored and tested positive for OSA, and the percentage of those who did not snore and tested positive for OSA. Prevalence was estimated from the proportion of screened patients.

Bivariable analysis (chi-square or Fisher exact test) was conducted to examine the association between demographic and clinical variables and snoring. Logistic regression was used to compare variables between the matched cohorts, and thus to determine factors independently associated with audible snoring.

\section{RESULTS}

Overall, 19 children were screened for eligibility in the study at the VCU Pediatric Dentistry Clinic from May to September 2015. Upon initiating dental treatment an attempt was made to reach a level of moderate sedation in which patients responded purposefully to verbal commands alone or with some light tactile stimulation. However, two children were unable to reach a sufficient level of sedation to be considered moderate. Therefore, snoring or nonsnoring under MS could not be accurately assessed/identified. These children were subsequently not accepted for further participation and could not be included in data analysis or any other diagnostic aspect of this study. From these children, 10 patients who snored (59\%) and 7 
control participants were recruited and completed the home monitoring portion of the study (Table 2). The 9 males and 8 females had an average age of 5.5 years (range $=4$ to 8$)$ and had an average BMI percentile of 59 (range $=2$ to 99th percentile). Sixty-five percent of the children were identified as African American $(n=11), 24 \%$ were Caucasian ( $n=4), 6 \%$ were Asian $(n=1)$, and one participant did not report ethnic affiliation. The snorers and nonsnorers had no differences in demographics.

\section{Table 2. Characteristics of study participants}

\begin{tabular}{llll} 
Variable & $\begin{array}{l}\text { Snorer } \\
(\mathrm{n}=10)\end{array}$ & $\begin{array}{l}\text { Nonsnorer } \\
(\mathrm{n}=7)\end{array}$ & $\begin{array}{l}\text { All } \\
\text { Participants }\end{array}$ \\
& $(58.8 \%)$ & $(41.1 \%)$ & \\
\hline Male & $5(50.0 \%)$ & $4(57.1 \%)$ & $9(52.9 \%)$ \\
Female & $5(50.0 \%)$ & $3(42.9 \%)$ & $8(47.1 \%)$ \\
AA & $6(60.0 \%)$ & $5(71.4 \%)$ & $11(64.7 \%)$ \\
Caucasian & $3(30.0 \%)$ & $1(14.3 \%)$ & $4(23.5 \%)$ \\
Asian & $1(10.0 \%)$ & $0(00.0 \%)$ & $1(5.9 \%)$ \\
UE & $0(00.0 \%)$ & $1(14.3 \%)$ & $1(5.9 \%)$
\end{tabular}

\section{AA, African American; UE: Unreported Ethnicity}

Table 3 depicts group comparisons using the outcomes measured in the study participants.

The primary outcome variable was the RDI indicator of OSA and although the children who snored during pediatric dental surgery had a nominally higher RDI than the control children who did not snore (mean $=5.3$ vs. 4.3 ), this difference was not statistically significant $(\mathrm{p}>0.7)$. Using a cutoff of RDI $>5$ to indicate OSA, 5 of 8 in the snorer group and 2 of 4 in the nonsnorer group met this criterion. Similar results were obtained using SI, and type 1 and type 2 snoring percentages in that specifically the snorers were nominally higher but results were statistically nonsignificant averages. "Type 1 and type 2 percentages" we recorded as the sum of the \% of type 1 and \% of type 2 snoring. In contrast, one nonsnoring participant had an oronasal sound with maximum relative loudness of $27 \mathrm{~dB}$, which resulted in the group mean being nominally higher than the snoring group's average.

The results from the questionnaires found a trend that both the PSQ and PSC average was 5 points higher in the snoring group than the average of the non-snorers though this difference was not statistically significant (PSQ $\mathrm{p}=$ 0.056 , PSC $\mathrm{p}>0.4$ ). The specific subscales for snoring, sleepiness, and behavior as described in the PSQ and PSC scores are strongly associated with SRBD. ${ }^{24}$

\section{Table 3. Comparing Sleep-study Measurements in Snorers and Non-snorers.}

Snoring N Mean SD P-value RDI

\begin{tabular}{ccccc}
\cline { 2 - 4 } Non-Snorer & 4 & 4.33 & 3.69 & 0.726 \\
Snorer & 8 & 5.33 & 4.86 &
\end{tabular}

Snoring Index (Snores/Hour)

\begin{tabular}{ccccc}
\cline { 2 - 4 } Non-Snorer & 3 & 158.33 & 226.68 & 0.314 \\
Snorer & 7 & 367.57 & 298.78 &
\end{tabular}

(\%) Type $1 \& 2$

\begin{tabular}{ccccc}
\cline { 2 - 4 } Non-Snorer & 3 & 32.67 & 56.58 & 0.210 \\
Snorer & 7 & 61.43 & 13.39 &
\end{tabular}

Oro Sound Max Rel. dB

\begin{tabular}{ccccc}
\cline { 2 - 4 } Non-Snorer & 3 & 10.00 & 14.80 & 0.395 \\
Snorer & 7 & 4.86 & 4.30 & \\
\hline \multicolumn{4}{c}{ PSQ }
\end{tabular}

\begin{tabular}{ccccc}
\cline { 2 - 4 } Non-Snorer & 7 & 3.86 & 5.15 & 0.056 \\
Snorer & 10 & 8.80 & 4.64 &
\end{tabular}

PSC

\begin{tabular}{ccccc}
\cline { 2 - 4 } Non-Snorer & 7 & 10.14 & 15.52 & 0.471 \\
Snorer & 10 & 15.30 & 13.15 &
\end{tabular}

\section{DISCUSSION}

One possible limitation to this study proved to be the ability to achieve an adequate level of sedation. Medications used during sedation aggravate OSA by altering the normal mechanism of respiration through decreasing pharyngeal muscle tone, depressing respiratory responses to hypoxia and hypercapnia; and in turn depressing arousal responses. ${ }^{19}$ In addition, pediatric patients undergoing MS are known in general to be at a higher risk of postoperative respiratory compromise, ${ }^{29}$ but those with undiagnosed OSA may be at even higher risk. 
In this study, all participants were sedated using various mixtures and dosages of chloral hydrate, midazolam, hydroxyzine and meperidine.

An additional potential limitation to this study was that capnographic monitoring was not used. This may have helped further explore the possible indicative relationship among OSA, pediatric sedation, $\mathrm{CO}_{2}$ retention, and snoring.

Overall, the relatively small number of snoring patients observed restricted the study power and limited the identification of potential confounding variables in predicting pediatric OSA. The incidence of snoring while under moderate sedation is likely to vary according to the study population and is potentially affected by the type of sedatives, the depth of sedation, and patient positioning.

In conclusion, although the difference in RDI of children who snored during pediatric dental surgery and those who did not snore was not statistically significant, the nominally higher RDI of the control children who snored is an interesting result that encourages further investigation with a larger sample size to possibly reduce many of the limitations. For this reason, the goal of this pilot study is to obtain information to perform a sample-size/power analysis for a larger study.

\section{ACKNOWLEDGMENTS}

This project was supported by Virginia Commonwealth University School of Dentistry Dental Research Fund.

\section{ABBREVIATIONS}

ASA: American Society of Anesthesiologists

BMI: Body Mass Index

IRB: Institutional Review Board

MS: Moderate Sedation

OSA: Obstructive Sleep Apnea

OSAS: Obstructive Sleep Apnea Syndrome

PSC: Pediatric Symptom Checklist

PSQ: Pediatric Sleep Questionnaire

RDI: Respiratory Disturbance Index

REDCap: Research Electronic Data Capture

RERA: Respiratory Effort Related Arousal

SI: Snoring Index

SRBD: Sleep-Related Breathing Disorders

\section{REFERENCES}

1. Pambianco DJ. Obstructive sleep apnea: can we gauge risk? Gastrointest Endosc. 2010; 71(7):1231-1232.

2. Clinical practice guideline: diagnosis and management of childhood obstructive sleep apnea syndrome. Section on Pediatric Pulmonology, Subcommittee on Obstructive Sleep Apnea Syndrome. American Academy of Pediatrics. et al. Pediatrics. 2002; 109(4):704 -712.

3. Lewandowski AS, Toliver-Sokol M, Palermo TM. Evidence-based review of subjective pediatric sleep measures. J Pediatr Psychol. 2011;36(7):780-793. administration of conscious sedation: previously undiagnosed obstructive sleep apnea. Ann Intern Med. 2003;139(8): 707-708.

University of Michigan. Sleep-Related Breathing Disorder Scale (SRBD Scale), From Pediatric Sleep Questionnaire, to Identify Symptoms of Obstructive Sleep Apnea in Children. http://inventions.umich.edu/technologies/3773_sleep-relatedbreathing-disorder-scale-srbd-scale-from-pediatric-sleepquestionnaire-to-identify-symptoms-of-obstructive-sleep-apnea-inchildren. Accessed on 01/13/2019.

23. Jellinek MS, Murphy JM, Little M, Pagano ME, Comer DM, Kelleher KJ. Use of the Pediatric Symptom Checklist (PSC) to screen for 
psychosocial problems in pediatric primary care: A national feasibility study. Arch Pediatr Adolesc Med. 1999;153(3):254-260.

24.

Chervin RD, Hedger KM, Dillon JE, Pituch KJ. Pediatric Sleep Questionnaire (PSQ): validity and reliability of scales for sleepdisordered breathing, snoring, sleepiness, and behavioral problems. Sleep Med. 2000;1(1):21-32.

25. University of Michigan. Pediatric Sleep Questionnaire - Designed as Research Screen for Symptoms of Obstructive Sleep Apnea and Other Sleep Disorders in Children. http://inventions.umich.edu/technologies/3766_pediatric-sleepquestionnaire-designed-as-research-screen-for-symptoms-ofobstructive-sleep-apnea-and-other-sleep-disorders-in-children. Accessed on 01/13/2019.

26. Fleming S, Thompson M, Stevens R, et al. Normal ranges of heart rate and respiratory rate in children from birth to 18 years of age: a systematic review of observational studies. Lancet. 2011;377(9770):1011-1018.

27. Su S1, Baroody FM, Kohrman M, Suskind D. A comparison of polysomnography and a portable home sleep study in the diagnosis of obstructive sleep apnea syndrome. Otolaryngol Head Neck Surg. 2004;131(6):844-850.

28. Michaelson PG1, Allan P, Chaney J, Mair EA. Validations of a portable home sleep study with twelve-lead polysomnography: comparisons and insights into a variable gold standard. Ann Otol Rhinol Laryngol. 2006;115(11):802-809.

29. Schechter MS. Technical report: diagnosis and management of childhood obstructive sleep apnea syndrome. Pediatrics. 2002; 109(4):e69.

\section{SUBMISSION \& CORRESPONDENCE INFORMATION}

\author{
Submitted for publication June 26, 2018 \\ Submitted in final revised form February 3, 2019 \\ Accepted for publication February 8, 2019
}

Address correspondence to: Harmeet K. Chiang, DDS, MS; School of Dentistry, Virginia Commonwealth University $4^{\text {th }}$ Floor Lyons Building, 520 N. $12^{\text {th }}$ Street, Box 980566, Richmond, VA 23298, Phone: 804-828-2977, Fax: 804827-2581, Email: hkchiang@vcu.edu

\section{DISCLOSURE STATEMENT}

Study data were collected and managed using REDCap (Research Electronic Data Capture) electronic data capture tools hosted at Virginia Commonwealth University (Harris PA, Taylor R, Thielke R, Payne J, Gonzalez N, Conde JG. Research electronic data capture (REDCap) - a metadata-driven methodology and workflow process for providing translational research informatics support. J Biomed Inform. 2009;42(2):377-381.). This was not an industry-supported study. The authors have indicated no financial conflicts of interest. 